\title{
Clinical and Laboratory Findings in Iranian Patients with Leukocyte Adhesion Deficiency (Study of 15 Cases)
}

\author{
Masoud Movahedi • Neda Entezari • Zahra Pourpak • \\ Setareh Mamishi • Zahra Chavoshzadeh • \\ Mohammad Gharagozlou • Bahram Mir-Saeeid-Ghazi • \\ Mohammad-Reza Fazlollahi • Fariborz Zandieh • \\ Mohammad-Hasan Bemanian • Aboulhasan Farhoudi • \\ Asghar Aghamohammadi
}

Published online: 18 September 2007

(C) Springer Science + Business Media, LLC 2007

Asghar Aghamohammadi (1) was inadvertently omitted from the list of authors. This paper was published in Vol. 27, No. 3, pp. 303-307.

Springer regrets this error.

\footnotetext{
The online version of the original article can be found at http://dx.doi. org/10.1007/s10875-006-9069-4.

M. Movahedi $\cdot$ N. Entezari $\cdot$ Z. Pourpak $\cdot$ M. Gharagozlou

M.-R. Fazlollahi · M.-H. Bemanian · A. Farhoudi •

A. Aghamohammadi

Immunology, Asthma and Allergy Research Institute,

Children Medical Center, Tehran University of Medical Sciences,

Tehran, IR, Iran

M. Movahedi $\cdot$ Z. Chavoshzadeh $\cdot$ M. Gharagozlou •

B. Mir-Saeeid-Ghazi · F. Zandieh · A. Farhoudi

Department of Immunology and Allergy,

Children Medical Center, Tehran University of Medical Sciences,

Tehran, IR, Iran

S. Mamishi

Department of Infections Disease, Children Medical Center,

Tehran University of Medical Sciences,

Tehran, IR, Iran

M. Movahedi ( $\square)$

Immunology, Asthma and Allergy Research Institute,

Children Medical Center, Tehran University of Medical Sciences,

No. 62, Gharib Street, Keshavarz Blvd, P. O. Box 14194, Tehran,

IR, Iran

e-mail: movahedm@sina.tums.ac.ir
} 